\title{
Memory Augmented Graph Neural Networks for Sequential Recommendation
}

\author{
Chen Ma, ${ }^{* 1}$ Liheng Ma, ${ }^{* 1,3}$ Yingxue Zhang, ${ }^{2}$ Jianing Sun, ${ }^{2}$ Xue Liu, ${ }^{1}$ Mark Coates ${ }^{1}$ \\ ${ }^{1}$ McGill University, ${ }^{2}$ Huawei Noah's Ark Lab in Montreal, ${ }^{3}$ Mila \\ \{chen.ma2, liheng.ma\}@mail.mcgill.ca, \{yingxue.zhang, jianing.sun\}@ huawei.com, \\ xueliu@cs.mcgill.ca, mark.coates@mcgill.ca
}

\begin{abstract}
The chronological order of user-item interactions can reveal time-evolving and sequential user behaviors in many recommender systems. The items that users will interact with may depend on the items accessed in the past. However, the substantial increase of users and items makes sequential recommender systems still face non-trivial challenges: (1) the hardness of modeling the short-term user interests; (2) the difficulty of capturing the long-term user interests; (3) the effective modeling of item co-occurrence patterns. To tackle these challenges, we propose a memory augmented graph neural network (MA-GNN) to capture both the long- and short-term user interests. Specifically, we apply a graph neural network to model the item contextual information within a short-term period and utilize a shared memory network to capture the long-range dependencies between items. In addition to the modeling of user interests, we employ a bilinear function to capture the co-occurrence patterns of related items. We extensively evaluate our model on five real-world datasets, comparing with several state-of-the-art methods and using a variety of performance metrics. The experimental results demonstrate the effectiveness of our model for the task of Top-K sequential recommendation.
\end{abstract}

\section{Introduction}

With the rapid growth of Internet services and mobile devices, personalized recommender systems play an increasingly important role in modern society. They can reduce information overload and help satisfy diverse service demands. Such systems bring significant benefits to at least two parties. They can: (i) help users easily discover products from millions of candidates, and (ii) create opportunities for product providers to increase revenue.

On the Internet, users access online products or items in a chronological order. The items a user will interact with in the future may depend strongly on the items he/she has accessed in the past. This property facilitates a practical application scenario-sequential recommendation. In the sequential recommendation task, in addition to the general user interest captured by all general recommendation models, we argue that there are three extra important factors to

\footnotetext{
${ }^{*}$ Work done as interns at Huawei Noah's Ark Lab in Montreal. Copyright (c) 2020, Association for the Advancement of Artificial Intelligence (www.aaai.org). All rights reserved.
}

model: user short-term interests, user long-term interests, and item co-occurrence patterns. The user short-term interest describes the user preference given several recently accessed items in a short-term period. The user long-term interest captures the long-range dependency between earlier accessed items and the items users will access in the future. The item co-occurrence pattern illustrates the joint occurrences of commonly related items, such as a mobile phone and a screen protector.

Although many existing methods have proposed effective models, we argue that they do not fully capture the aforementioned factors. First, methods like Caser (Tang and Wang 2018), MARank (Yu et al. 2019), and Fossil (He and McAuley 2016a) only model the short-term user interest and ignore the long-term dependencies of items in the item sequence. The importance of capturing the long-range dependency has been confirmed by (Belletti, Chen, and Chi 2019). Second, methods like SARSRec (Kang and McAuley 2018) do not explicitly model the user short-term interest. Neglecting the user short-term interest prevents the recommender system from understanding the time-varying user intention over a short-term period. Third, methods like GC-SAN (Xu et al. 2019) and GRU4Rec+ (Hidasi and Karatzoglou 2018) do not explicitly capture the item co-occurrence patterns in the item sequences. Closely related item pairs often appear one after the other and a recommender system should take this into account.

To incorporate the factors mentioned above, we propose a memory augmented graph neural network (MA-GNN) to tackle the sequential recommendation task. This consists of a general interest module, a short-term interest module, a long-term interest module, and an item co-occurrence module. In the general interest module, we adopt a matrix factorization term to model the general user interest without considering the item sequential dynamics. In the short-term interest module, we aggregate the neighbors of items using a GNN to form the user intentions over a short period. These can capture the local contextual information and structure (Battaglia et al. 2018) within this short-term period. To model the long-term interest of users, we use a shared key-value memory network to generate the interest representations based on users' long-term item sequences. By doing this, other users with similar preferences will be taken into consideration when recommending an item. To combine 
the short-term and long-term interest, we introduce a gating mechanism in the GNN framework, which is similar to the long short-term memory (LSTM) (Hochreiter and Schmidhuber 1997). This controls how much the long-term or the short-term interest representation can contribute to the combined representation. In the item co-occurrence module, we apply a bilinear function to capture the closely related items that appear one after the other in the item sequence. We extensively evaluate our model on five real-world datasets, comparing it with many state-of-the-art methods using a variety of performance validation metrics. The experimental results not only demonstrate the improvements of our model over other baselines but also show the effectiveness of the proposed components.

To summarize, the major contributions of this paper are:

- To model the short-term and long-term interests of users, we propose a memory augmented graph neural network to capture items' short-term contextual information and long-range dependencies.

- To effectively fuse the short-term and long-term interests, we incorporate a gating mechanism within the GNN framework to adaptively combine these two kinds of hidden representations.

- To explicitly model the item co-occurrence patterns, we use a bilinear function to capture the feature correlations between items.

- Experiments on five real-world datasets show that the proposed MA-GNN model significantly outperforms the state-of-the-art methods for sequential recommendation.

\section{Related Work \\ General Recommendation}

Early recommendation studies largely focused on explicit feedback (Koren 2008). The recent research focus is shifting towards implicit data (Tran et al. 2019; Li and She 2017). Collaborative filtering (CF) with implicit feedback is usually treated as a Top-K item recommendation task, where the goal is to recommend a list of items to users that users may be interested in. It is more practical and challenging (Pan et al. 2008), and accords more closely with the real-world recommendation scenario. Early works mostly rely on matrix factorization techniques (Hu, Koren, and Volinsky 2008; Rendle et al. 2009) to learn latent features of users and items. Due to their ability to learn salient representations, (deep) neural network-based methods (He et al. 2017) are also adopted. Autoencoder-based methods (Ma et al. 2018; 2019) have also been proposed for Top-K recommendation. In (Lian et al. 2018; Xue et al. 2017), deep learning techniques are used to boost the traditional matrix factorization and factorization machine methods.

\section{Sequential Recommendation}

The sequential recommendation task takes as input the chronological item sequence. A Markov chain (Cheng et al. 2013) is a classical option for modelling the data. For example, FPMC (Rendle, Freudenthaler, and Schmidt-Thieme 2010) factorizes personalized Markov chains in order to capture long-term preferences and short-term transitions. Fossil (He and McAuley 2016a) combines similarity-based models with high-order Markov chains. TransRec (He, Kang, and McAuley 2017) proposes a translation-based method for sequential recommendation. Recently, inspired by the advantages of sequence learning in natural language processing, researchers have proposed (deep) neural network based methods to learn the sequential dynamics. For instance, Caser (Tang and Wang 2018) applies a convolutional neural network (CNN) to process the item embedding sequence. Recurrent neural network (RNN)-based methods, especially gated recurrent unit (GRU)-based methods (Hidasi and Karatzoglou 2018; Hidasi et al. 2016; Li et al. 2017) have been used to model the sequential patterns for the task of session-based recommendation. Self-attention (Vaswani et al. 2017) exhibits promising performance in sequence learning and is starting to be used in sequential recommendation. SASRec (Kang and McAuley 2018) leverages self-attention to adaptively take into account the interactions between items. Memory networks (Chen et al. 2018; Huang et al. 2018) are also adopted to memorize the items that will play a role in predicting future user actions.

However, our proposed model is different from previous models. We apply a graph neural network with external memories to capture the short-term item contextual information and long-term item dependencies. In addition, we also incorporate an item co-occurrence module to model the relationships between closely related items.

\section{Problem Formulation}

The recommendation task considered in this paper takes sequential implicit feedback as training data. The user preference is represented by a user-item sequence in chronological order, $\mathcal{S}^{u}=\left(I_{1}, I_{2}, \ldots, I_{\left|\mathcal{S}^{u}\right|}\right)$, where $I_{*}$ are item indexes that user $u$ has interacted with. Given the earlier subsequence $\mathcal{S}_{1: t}^{u}\left(t<\left|\mathcal{S}^{u}\right|\right)$ of $M$ users, the problem is to recommend a list of $K$ items from a total of $N$ items $(K<N)$ to each user and evaluate whether the items in $\mathcal{S}_{t+1:\left|\mathcal{S}^{u}\right|}^{u}$ appear in the recommended list.

\section{Methodology}

In this section, we introduce the proposed model, MA-GNN, which applies a memory augmented graph neural network for the sequential recommendation task. We introduce four factors that have an impact on the user preference and intention learning. Then we introduce the prediction and training procedure of the proposed model.

\section{General Interest Modeling}

The general or static interest of a user captures the inherent preferences of the user and is assumed to be stable over time. To capture the general user interest, we employ a matrix factorization term without considering the sequential dynamics of items. This term takes the form

$$
\mathbf{p}_{u}^{\top} \cdot \mathbf{q}_{j},
$$

where $\mathbf{p}_{u} \in \mathbb{R}^{d}$ is the embedding of user $u, \mathbf{q}_{j} \in \mathbb{R}^{d}$ is the output embedding of item $j$, and $d$ is the dimension of the latent space. 


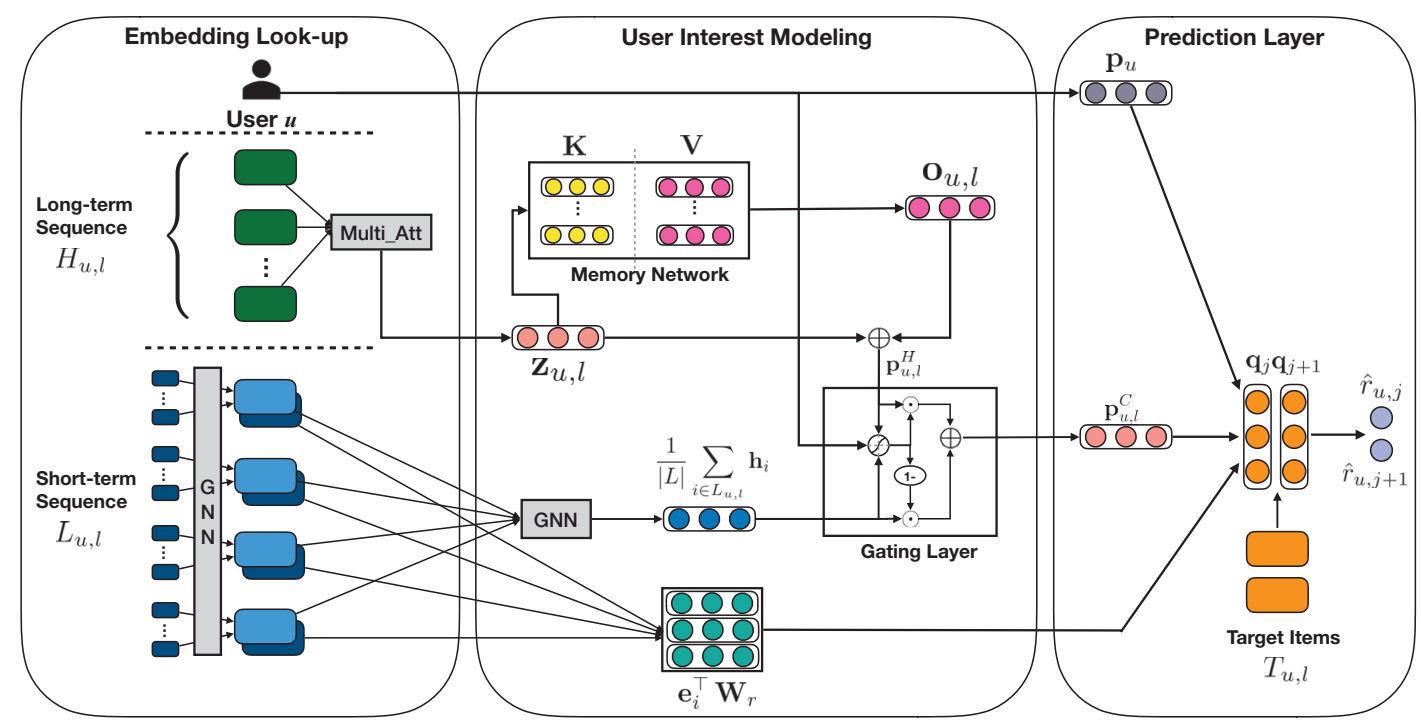

Figure 1: The model architecture of MA-GNN. $\oplus$ denotes element-wise addition and $\odot$ denotes element-wise multiplication.

\section{Short-term Interest Modeling}

A user's short-term interest describes the user's current preference and is based on several recently accessed items in a short-term period. The items a user will interact with in the near future are likely to be closely related to the items she just accessed, and this property of user behaviors has been confirmed in many previous works (Tang and Wang 2018; Hidasi and Karatzoglou 2018; He and McAuley 2016a). Therefore, it is very important in sequential recommendation to effectively model the user's short-term interest, as reflected by recently accessed items.

To explicitly model the user short-term interest, we conduct a sliding window strategy to split the item sequence into fine-grained sub-sequences. We can then focus on the recent sub-sequence to predict which items will appear next and ignore the irrelevant items that have less impact. For each user $u$, we extract every $|L|$ successive items as input and their next $|T|$ items as the targets to be predicted, where $L_{u, l}=\left(I_{l}, I_{l+1}, \ldots, I_{l+|L|-1}\right)$ is the $l$-th sub-sequence of user $u$. Then the problem can be formulated as: in the useritem interaction sequence $\mathcal{S}^{u}$, given a sequence of $|L|$ successive items, how likely is it that the predicted items accord with the target $|T|$ items for that user. Due to their ability to perform neighborhood information aggregation and local structure learning (Battaglia et al. 2018), graph neural networks (GNNs) are a good match for the task of aggregating the items in $L_{u, l}$ to learn user short-term interests.

Item Graph Construction. Since item sequences are not inherently graphs for GNN training, we need to build a graph to capture the connections between items. For each item in item sequences, we extract several subsequent items (three items in our experiments) and add edges between them. We perform this for each user and count the number of edges of extracted item pairs across all users. Then we row-normalize the adjacency matrix. As such, relevant items that appear closer to one another in the sequence can be extracted. An example of how to extract item neighbors and build the adjacency matrix is shown in Figure 2. We denote the extracted adjacency matrix as $\mathbf{A}$, where $A_{i, k}$ denotes the normalized node weight of item $k$ regarding item $i$. And the neighboring items of item $i$ is denoted as $\mathcal{N}_{i}$.

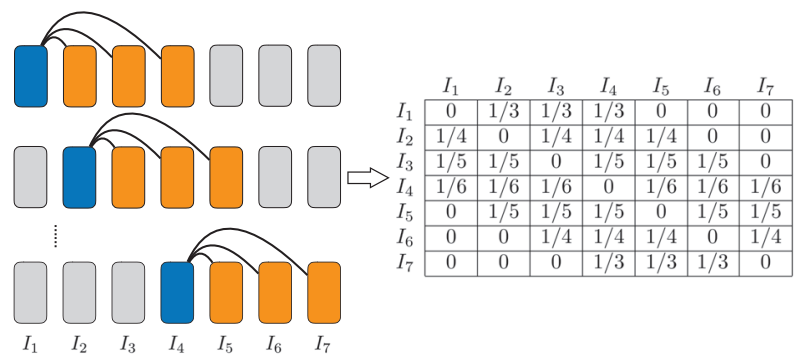

Figure 2: Item adjacent matrix construction example.

Short-term Interest Aggregation. To capture the user short-term interest, we use a two-layer GNN to aggregate the neighboring items in $L_{u, l}$ for learning the user shortterm interest representation. Formally, for an item $j$ in the $l$-th short-term window $L_{u, l}$, its input embedding is represented as $\mathbf{e}_{j} \in \mathbb{R}^{d}$. The user short-term interest is then:

$$
\begin{gathered}
\mathbf{h}_{i}=\tanh \left(\mathbf{W}^{(1)} \cdot\left[\sum_{k \in \mathcal{N}_{i}} \mathbf{e}_{k} A_{i, k} ; \mathbf{e}_{i}\right]\right), \forall i \in L_{u, l}, \\
\mathbf{p}_{u, l}^{S}=\tanh \left(\mathbf{W}^{(2)} \cdot\left[\frac{1}{|L|} \sum_{i \in L_{u, l}} \mathbf{h}_{i} ; \mathbf{p}_{u}\right]\right),
\end{gathered}
$$

where $[\cdot ; \cdot] \in \mathbb{R}^{2 d}$ denotes vertical concatenation, $\mathbf{W}^{(1)}, \mathbf{W}^{(2)} \in \mathbb{R}^{d \times 2 d}$ are the learnable parameters in the graph neural network, and the superscript $S$ denotes that the representation is from the user short-term interest. By aggregating neighbors of items in $L_{u, l}, \mathbf{p}_{u, l}^{S}$ represents a unionlevel summary (Tang and Wang 2018; Yu et al. 2019) indicating which items are closely relevant to the items in $L_{u, l}$. 
Based on the summarized user short-term interest, the items that a user will access next can be inferred.

However, directly applying the above GNN to make predictions clearly neglects the long-term user interest in the past $H_{u, l}=\left(I_{1}, I_{2}, \ldots, I_{l-1}\right)$. There may be some items outside the short-term window $L_{u, l}$ that can express the user preference or indicate the user state. These items can play an important role in predicting items that will be accessed in the near future. This long-term dependency has been confirmed in many previous works (Liu et al. 2018; Xu et al. 2019; Belletti, Chen, and Chi 2019). Thus, how to model the longterm dependency and balance it with the short-term context is a crucial question in sequential recommendation.

\section{Long-term Interest Modeling}

To capture the long-term user interest, we can use external memory units (Sukhbaatar et al. 2015; Zhang et al. 2017) to store the time-evolving user interests given the user accessed items in $H_{u, l}=\left(I_{1}, I_{2}, \ldots, I_{l-1}\right)$. However, maintaining the memory unit for each user has a huge memory overhead to store the parameters. Meanwhile, the memory unit may capture information that is very similar to that represented by the user embedding $\mathbf{p}_{u}$. Therefore, we propose to use a memory network to store the latent interest representation shared by all users, where each memory unit represents a certain type of latent user interest, such as the user interest regarding different categories of movies. Given the items accessed by a user in the past $H_{u, l}$, we can learn a combination of different types of interest to reflect the user long-term interest (or state) before $L_{u, l}$.

Instead of performing a summing operation to generate the query as in the original memory network (Sukhbaatar et al. 2015), we apply a multi-dimensional attention model to generate the query embedding. This allows discriminating informative items that can better reflect the user preference to have a greater influence on the positioning of the corresponding external memory units. Formally, we denote the item embeddings in $H_{u, l}$ as $\mathbf{H}_{u, l} \in \mathbb{R}^{d \times\left|H_{u, l}\right|}$. The multidimensional attention to generate the query embedding $\mathbf{z}_{u, l}$ is computed as:

$$
\begin{aligned}
\mathbf{H}_{u, l} & :=\mathbf{H}_{u, l}+\operatorname{PE}\left(H_{u, l}\right), \\
\mathbf{S}_{u, l}=\operatorname{softmax} & \left(\mathbf{W}_{a}^{(3)} \tanh \left(\mathbf{W}_{a}^{(1)} \mathbf{H}_{u, l}+\left(\mathbf{W}_{a}^{(2)} \mathbf{p}_{u}\right) \otimes \mathbf{1}_{\phi}\right)\right), \\
\mathbf{Z}_{u, l} & =\tanh \left(\mathbf{S}_{u, l} \cdot \mathbf{H}_{u, l}^{\top}\right), \\
\mathbf{z}_{u, l} & =\operatorname{avg}\left(\mathbf{Z}_{u, l}\right),
\end{aligned}
$$

where $\mathrm{PE}(\cdot)$ is the sinusoidal positional encoding function that maps the item positions into position embeddings, which is the same as the one used in Transformer (Vaswani et al. 2017). $\phi$ equals to $\left|H_{u, l}\right|, \otimes$ denotes the outer product. $\mathbf{W}_{a}^{(1)}, \mathbf{W}_{a}^{(2)} \in \mathbb{R}^{d \times d}$ and $\mathbf{W}_{a}^{(3)} \in \mathbb{R}^{h \times d}$ are the learnable parameters in the attention model, and $h$ is the hyperparameter to control the number of dimensions in the attention model. $\mathbf{S}_{u, l} \in \mathbb{R}^{h \times\left|H_{u, l}\right|}$ is the attention score matrix. $\mathbf{Z}_{u, l} \in \mathbb{R}^{h \times d}$ is the matrix representation of the query, and each of the $h$ rows represents a different aspect of the query.
Finally, $\mathbf{z}_{u, l} \in \mathbb{R}^{d}$ is the combined query embedding that averages the different aspects.

Given the query embedding $\mathbf{z}_{u, l}$, we use this query to find the appropriate combination of the shared user latent interest in the memory network. Formally, the keys and values of the memory network (Sukhbaatar et al. 2015; Miller et al. 2016) are denoted as $\mathbf{K} \in \mathbb{R}^{d \times m}$ and $\mathbf{V} \in$ $\mathbb{R}^{d \times m}$, respectively, where $m$ is the number of memory units in the memory network. Therefore, the user long-term interest embedding can be modeled as:

$$
\begin{aligned}
& s_{i}=\operatorname{softmax}\left(\mathbf{z}_{u, l}^{\top} \cdot \mathbf{k}_{i}\right), \\
& \mathbf{o}_{u, l}=\sum_{i} s_{i} \mathbf{v}_{i} \\
& \mathbf{p}_{u, l}^{H}=\mathbf{z}_{u, l}+\mathbf{o}_{u, l},
\end{aligned}
$$

where $\mathbf{k}_{i}, \mathbf{v}_{i} \in \mathbb{R}^{d}$ are the $i$-th memory unit and the superscript $H$ denotes the representation is from the user longterm interest.

\section{Interest Fusion}

We have obtained the user short-term interest representation and the long-term interest representation. The next aim is to combine these two kinds of hidden representations in the GNN framework to facilitate the user preference prediction on unrated items. Here, we modify Eq. 2 to bridge the user short-term interest and long-term interest.

Specifically, we borrow the idea of LSTM (Hochreiter and Schmidhuber 1997) that uses learnable gates to balance the current inputs and historical hidden states. Similarly, we propose a learnable gate to control how much the recent user interest representation and the long-term user interest representation can contribute to the combined user interest for item prediction:

$$
\begin{aligned}
& \mathbf{g}_{u, l}=\sigma\left(\mathbf{W}_{g}^{(1)} \cdot \frac{1}{|L|} \sum_{i \in L_{u, l}} \mathbf{h}_{i}+\mathbf{W}_{g}^{(2)} \cdot \mathbf{p}_{u, l}^{H}+\mathbf{W}_{g}^{(3)} \cdot \mathbf{p}_{u}\right) \\
& \mathbf{p}_{u, l}^{C}=\mathbf{g}_{u, l} \odot \frac{1}{|L|} \sum_{i \in L_{u, l}} \mathbf{h}_{i}+\left(\mathbf{1}_{d}-\mathbf{g}_{u, l}\right) \odot \mathbf{p}_{u, l}^{H}
\end{aligned}
$$

where $\mathbf{W}_{g}^{(1)}, \mathbf{W}_{g}^{(2)}, \mathbf{W}_{g}^{(3)} \in \mathbb{R}^{d \times d}$ are the learnable parameters in the gating layer, $\odot$ denotes the element-wise multiplication, and $\mathbf{g}_{u, l} \in \mathbb{R}^{d}$ is the learnable gate. The superscript $C$ denotes the fusion of long- and short-term interests.

\section{Item Co-occurrence Modeling}

Successful learning of pairwise item relationships is a key component of recommender systems due to its effectiveness and interpretability. This has been studied and exploited in many recommendation models (Deshpande and Karypis 2004; Ning, Desrosiers, and Karypis 2015). In the sequential recommendation problem, the closely related items may appear one after another in the item sequence. For example, after purchasing a mobile phone, the user is much more likely to buy a mobile phone case or protector. To capture the item co-occurrence patterns, we use a bilinear function 
to explicitly model the pairwise relations between the items in $L_{u, l}$ and other items. This function takes the form

$$
\mathbf{e}_{i}^{\top} \mathbf{W}_{r} \mathbf{q}_{j}
$$

where $\mathbf{W}_{r} \in \mathbb{R}^{d \times d}$ is a matrix of the learnable parameters that captures the correlations between item latent features.

\section{Prediction and Training}

To infer the user preference, we have a prediction layer to combine the aforementioned factors together:

$$
\hat{r}_{u, j}=\mathbf{p}_{u}^{\top} \cdot \mathbf{q}_{j}+\mathbf{p}_{u, l}^{C \top} \cdot \mathbf{q}_{j}+\frac{1}{|L|} \sum_{i \in L_{u, l}} \mathbf{e}_{i}^{\top} \mathbf{W}_{r} \mathbf{q}_{j} .
$$

As the training data is derived from the user implicit feedback, we optimize the proposed model with respect to the Bayesian Personalized Ranking objective (Rendle et al. 2009) via gradient descent. This involves optimizing the pairwise ranking between the positive (observed) and negative (non-observed) items:

$$
\begin{array}{r}
\underset{\mathbf{U}, \mathbf{Q}, \mathbf{E}, \boldsymbol{\Theta}}{\arg \min } \sum_{u} \sum_{l} \sum_{\left(L_{u, l}, H_{u, l}, j, k\right)}-\log \sigma\left(\hat{r}_{u, j}-\hat{r}_{u, k}\right)+ \\
\lambda\left(\|\mathbf{P}\|^{2}+\|\mathbf{Q}\|^{2}+\|\mathbf{E}\|^{2}+\|\mathbf{\Theta}\|^{2}\right) .
\end{array}
$$

Here $j$ denotes the positive item in $T_{u, l}$, and $k$ denotes the randomly sampled negative item, $\sigma$ is the sigmoid function, $\Theta$ denotes other learnable parameters in the model, and $\lambda$ is the regularization parameter. $\mathbf{p}_{*}, \mathbf{q}_{*}$ and $\mathbf{e}_{*}$ are column vectors of $\mathbf{P}, \mathbf{Q}$ and $\mathbf{E}$, respectively. When minimizing the objective function, the partial derivatives w.r.t. all parameters are computed by gradient descent with back-propagation.

\section{Evaluation}

In this section, we first describe the experimental set-up. We then report the results of conducted experiments and demonstrate the effectiveness of the proposed modules.

\section{Datasets}

The proposed model is evaluated on five real-world datasets from various domains with different sparsities: MovieLens$20 M$ (Harper and Konstan 2016), Amazon-Books and Amazon-CDs (He and McAuley 2016b), GoodreadsChildren and Goodreads-Comics (Wan and McAuley 2018). MovieLens-20M is a user-movie dataset collected from the MovieLens website; the dataset has 20 million user-movie interactions. The Amazon-Books and Amazon-CDs datasets are adopted from the Amazon review dataset with different categories, i.e., CDs and Books, which cover a large amount of user-item interaction data, e.g., user ratings and reviews. The Goodreads-Children and Goodreads-Comics datasets were collected in late 2017 from the goodreads website with a focus on the genres of Children and Comics. In order to be consistent with the implicit feedback setting, we keep those with ratings no less than four (out of five) as positive feedback and treat all other ratings as missing entries on all datasets. To filter noisy data, we only keep the users with at least ten ratings and the items with at least ten ratings. The data statistics after preprocessing are shown in Table 1.
For each user, we use the earliest $70 \%$ of the interactions in the user sequence as the training set and use the next $10 \%$ of interactions as the validation set for hyper-parameter tuning. The remaining $20 \%$ constitutes the test set for reporting model performance. Note that during the testing procedure, the input sequences include the interactions in both the training set and validation set. The learning of all the models is carried out five times to report the average results.

Table 1: The statistics of the datasets.

\begin{tabular}{|c|c|c|c|c|}
\hline Dataset & \#Users & \#Items & \#Interactions & Density \\
\hline CDs & 17,052 & 35,118 & 472,265 & $0.079 \%$ \\
\hline Books & 52,406 & 41,264 & $1,856,747$ & $0.086 \%$ \\
\hline Children & 48,296 & 32,871 & $2,784,423$ & $0.175 \%$ \\
\hline Comics & 34,445 & 33,121 & $2,411,314$ & $0.211 \%$ \\
\hline ML20M & 129,797 & 13,649 & $9,921,393$ & $0.560 \%$ \\
\hline
\end{tabular}

\section{Evaluation Metrics}

We evaluate all the methods in terms of Recall@ $K$ and $N D C G @ K$. For each user, Recall@K (R@K) indicates what percentage of her rated items emerge in the top $K$ recommended items. NDCG@K $(\mathrm{N} @ \mathrm{~K})$ is the normalized discounted cumulative gain at $K$, which takes the position of correctly recommended items into account. $K$ is set to 10 .

\section{Methods Studied}

To demonstrate the effectiveness of our model, we compare to the following recommendation methods: (1) BPRMF, Bayesian Personalized Ranking based Matrix Factorization (Rendle et al. 2009), a classic method for learning pairwise item rankings; (2) GRU4Rec, Gated Recurrent Unit for Recommendation (Hidasi et al. 2016), which uses recurrent neural networks to model item sequences for sessionbased recommendation; (3) GRU4Rec+, an improved version of GRU4Rec (Hidasi and Karatzoglou 2018), which adopts an advanced loss function and sampling strategy; (4) GC-SAN, Graph Contextualized Self-Attention Network (Xu et al. 2019), which uses a graph neural network and self-attention mechanism for session-based recommendation; (5) Caser, Convolutional Sequence Embedding Recommendation, (Tang and Wang 2018), which captures high-order Markov chains via convolution operations; (6) SASRec, Self-Attention based Sequential Recommendation (Kang and McAuley 2018), which uses an attention mechanism to identify relevant items for prediction; (7) MARank, Multi-order Attentive Ranking model (Yu et al. 2019), which unifies individual- and union-level item interactions to infer user preference from multiple views; (8) MA-GNN, the proposed model, which applies a memory augmented GNN to combine the recent and historical user interests and adopts a bilinear function to explicitly capture the item-item relations.

\section{Experiment Settings}

In the experiments, the latent dimension of all the models is set to 50. For the session-based methods, we treat the items 
Table 2: The performance comparison of all methods in terms of Recall@10 and NDCG@10. The best performing method is boldfaced. The underlined number is the second best performing method. $*$ indicates the statistical significance for $p<=0.01$ compared to the best baseline method based on the paired t-test.

\begin{tabular}{|l|cc|cc|cc|cc|cc|}
\hline \multirow{2}{*}{ Methods } & \multicolumn{2}{|c|}{ CDs } & \multicolumn{2}{|c|}{ Books } & \multicolumn{2}{c|}{ Children } & \multicolumn{2}{c|}{ Comics } & \multicolumn{2}{c|}{ ML20M } \\
\cline { 2 - 11 } & $\mathrm{R} @ 10$ & $\mathrm{~N} @ 10$ & $\mathrm{R} @ 10$ & $\mathrm{~N} @ 10$ & $\mathrm{R} @ 10$ & $\mathrm{~N} @ 10$ & $\mathrm{R} @ 10$ & $\mathrm{~N} @ 10$ & $\mathrm{R} @ 10$ & $\mathrm{~N} @ 10$ \\
\hline BPRMF & 0.0269 & 0.0145 & 0.0260 & 0.0151 & 0.0814 & 0.0664 & 0.0788 & 0.0713 & 0.0774 & 0.0785 \\
\hline GRU4Rec & 0.0302 & 0.0154 & 0.0266 & 0.0157 & 0.0857 & 0.0715 & 0.0958 & 0.0912 & 0.0804 & 0.0912 \\
GRU4Rec+ & 0.0356 & 0.0171 & 0.0301 & 0.0171 & 0.0978 & 0.0821 & 0.1288 & 0.1328 & 0.0904 & 0.0946 \\
GC-SAN & 0.0372 & $\underline{0.0196}$ & 0.0344 & $\underline{0.0256}$ & 0.1099 & 0.0967 & 0.1490 & 0.1563 & 0.1161 & $\underline{0.1119}$ \\
\hline Caser & 0.0297 & 0.0163 & 0.0297 & 0.0216 & 0.1060 & 0.0943 & 0.1473 & 0.1529 & $\underline{0.1169}$ & 0.1116 \\
SARSRec & 0.0341 & 0.0193 & $\underline{0.0358}$ & 0.0240 & $\underline{0.1165}$ & $\underline{0.1007}$ & $\underline{0.1494}$ & $\underline{0.1592}$ & 0.1069 & 0.1014 \\
MARank & $\underline{0.0382}$ & 0.0151 & 0.0355 & 0.0223 & 0.1092 & 0.0980 & 0.1325 & 0.1431 & 0.1002 & 0.1031 \\
\hline MA-GNN & $\mathbf{0 . 0 4 4 2} *$ & $\mathbf{0 . 0 2 1 4}$ & $\mathbf{0 . 0 4 3 2} *$ & $\mathbf{0 . 0 2 7 9 *}$ & $\mathbf{0 . 1 2 1 5}$ & $\mathbf{0 . 1 1 3 7} *$ & $\mathbf{0 . 1 6 1 7} *$ & $\mathbf{0 . 1 6 5 5}$ & $\mathbf{0 . 1 2 3 6}$ & $\mathbf{0 . 1 2 7 2} *$ \\
Improvement & $15.7 \%$ & $9.2 \%$ & $20.7 \%$ & $9.0 \%$ & $4.3 \%$ & $12.9 \%$ & $8.23 \%$ & $4.0 \%$ & $5.7 \%$ & $13.7 \%$ \\
\hline
\end{tabular}

in a short-term window as one session. For GRU4Rec and GRU4Rec+, we find that a learning rate of 0.001 and batch size of 50 can achieve good performance. These two methods adopt Top1 loss and BPR-max loss, respectively. For GC-SAN, we set the weight factor $\omega$ to 0.5 and the number of self-attention blocks $k$ to 4 . For Caser, we follow the settings in the author-provided code to set $|L|=5,|T|=3$, the number of horizontal filters to 16 , and the number of vertical filters to 4 . For SASRec, we set the number of selfattention blocks to 2 , the batch size to 128 , and the maximum sequence length to 50. For MARank, we follow the original paper to set the number of depending items as 6 and the number of hidden layers as 4 . The network architectures of the above methods are configured to be the same as described in the original papers. The hyper-parameters are tuned on the validation set.

For MA-GNN, we follow the same setting in Caser to set $|L|=5$ and $|T|=3$. Hyper-parameters are tuned by grid search on the validation set. The embedding size $d$ is also set to 50. The value of $h$ and $m$ are selected from $\{5,10,15,20\}$. The learning rate and $\lambda$ are set to 0.001 and 0.001 , respectively. The batch size is set to 4096 .

\section{Performance Comparison}

The performance comparison results are shown in Table 2.

Observations about our model. First, the proposed model, MA-GNN, achieves the best performance on all five datasets with all evaluation metrics, which illustrates the superiority of our model. Second, MA-GNN outperforms SASRec. Although SASRec adopts the attention model to distinguish the items users have accessed, it neglects the common item co-occurrence patterns between two closely related items, which is captured by our bilinear function. Third, MA-GNN achieves better performance than Caser, GC-SAN and MARank. One major reason is that these three methods only model the user interests in a short-term window or session, but fail to capture the long-term item dependencies. On the contrary, we have a memory network to generate the long-term user interest. Fourth, MA-GNN obtains better results than GRU4Rec and GRU4Rec+. One possible reason is that GRU4Rec and GRU4Rec+ are session-based methods that do not explicitly model the user general inter- ests. Fifth, MA-GNN outperforms BPRMF. BPRMF only captures the user general interests, and does not incorporate the sequential patterns of user-item interactions. As such, BPRMF fails to capture the user short-term interests.

Other observations. First, all the results reported on MovieLens-20M, GoodReads-Children and GoodReadsComics are better than the results on other datasets. The major reason is that the other datasets are sparser and data sparsity negatively impacts recommendation performance. Second, MARank, SASRec and GC-SAN outperform Caser on most of the datasets. The main reason is that these methods can adaptively measure the importance of different items in the item sequence, which may lead to more personalized user representation learning. Third, Caser achieves better performance than GRU4Rec and GRU4Rec+ in most cases. One possible reason is that Caser explicitly inputs the user embeddings into its prediction layer, which allows it to learn general user interests. Fourth, GRU4Rec+ performs better than GRU4Rec on all datasets. The reason is that GRU4Rec+ not only captures the sequential patterns in the user-item sequence but also has a superior objective function-BPR-max. Fifth, all the methods perform better than BPR. This illustrates that a technique that can only perform effective modeling of the general user interests is incapable of adequately capturing the user sequential behavior.

Table 3: The ablation analysis. $S$ denotes the short-term interest module, $H$ denotes the long-term interest module, concat denotes the concatenation operation.

\begin{tabular}{|l|c|c|c|c|}
\hline \multirow{2}{*}{ Architecture } & \multicolumn{2}{|c|}{ CDs } & \multicolumn{2}{c|}{ Books } \\
\cline { 2 - 5 } & R@10 & N@10 & R@ 10 & N@ 10 \\
\hline (1) MF & 0.0269 & 0.0145 & 0.0310 & 0.0177 \\
(2) MF+S & 0.0306 & 0.0158 & 0.0324 & 0.0185 \\
(3) MF+S+H+gating & 0.0401 & 0.0191 & 0.0351 & 0.0208 \\
(4) MF+S+H+concat & 0.0295 & 0.0148 & 0.0296 & 0.0206 \\
(5) MF+S+H+GRU & 0.0268 & 0.0147 & 0.0306 & 0.0204 \\
(6) MA-GNN & $\mathbf{0 . 0 4 4 2}$ & $\mathbf{0 . 0 2 1 4}$ & $\mathbf{0 . 0 4 3 2}$ & $\mathbf{0 . 0 2 7 9}$ \\
\hline
\end{tabular}




\section{Ablation Analysis}

To verify the effectiveness of the proposed short-term interest modeling module, long-term interest modeling module, and item co-occurrence modeling module, we conduct an ablation study in Table 3 . This demonstrates the contribution of each module to the MA-GNN model. In (1), we utilize only the BPR matrix factorization without other components to show the performance of modeling user general interests. In (2), we incorporate the user short-term interest by the vanilla graph neural network (Eq. 1 and 2) on top of (1). In (3), we integrate the user long-term interest with the short-term interest via the proposed interest fusion module (Eq. 3, 4 and 5) on top of (2). In (4), we replace the interest fusion module in (3) with the concatenation operation to link the short-term interest and long-term interest. In (5), we replace the concatenation operation with a gated recurrent unit (Cho et al. 2014) (GRU). In (6), we present the overall MA-GNN model to show the effectiveness of the item co-occurrence modeling module.

From the results shown in Table 3, we make the following observations. First, comparing (1) and (2)-(6), we can observe that although the conventional BPR matrix factorization can capture the general user interests, it cannot effectively model the short-term user interests. Second, from (1) and (2), we observe that incorporating the short-term interest using the conventional aggregation function of the GNN slightly improves the model performance. Third, in (3), (4) and (5), we compare three ways to bridge the user short-term interest and long-term interest. From the results, we can observe that our proposed gating mechanism achieves considerably better performance than concatenation or the GRU, which demonstrates that our gating mechanism can adaptively combine these two kinds of hidden representations. Fourth, from (3) and (6), we observe that by incorporating the item co-occurrence pattern, the performance further improves. The results show the effectiveness of explicitly modeling the co-occurrence patterns of the items that a user has accessed and those items that the user may interact with in the future. The item co-occurrence pattern can provide a significant amount of supplementary information to help capture the user sequential dynamics.

\section{Influence of Hyper-parameters}

The dimension $h$ of the multi-dimensional attention model and the number $m$ of the memory units are two important hyper-parameters in the proposed model. We investigate their effects on CDs and Comics datasets in Figure 3.

From the results in Figure 3, we observe that both the multi-dimensional attention and the memory network contribute to capturing the long-term user interests. These two components lead to a larger improvement in performance for the CDs dataset compared to the Comics dataset, indicating that they may help to alleviate the data sparsity problem.

\section{Memory Visualization}

To validate whether each memory unit can represent a certain type of user interests, we conduct a case study on the MovieLens dataset to verify how each memory unit functions given different movies. We randomly select a user and

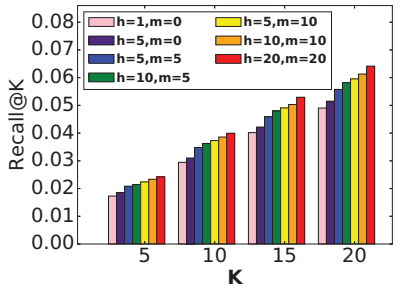

(a) $h$ and $m$ on CDs

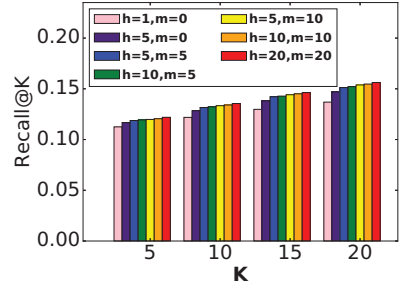

(b) $h$ and $m$ on Comics
Figure 3: The variation of $h$ and $m$.

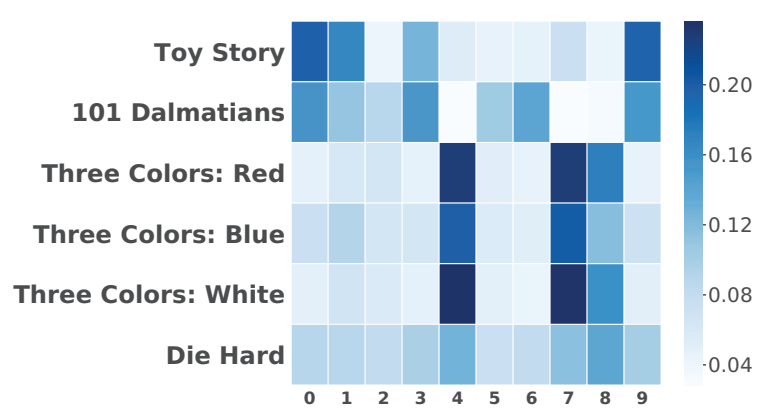

Figure 4: The attention visualization of memory networks.

several movies she watched. For simplicity, we treat each selected item as a query to visualize the attention weight $s_{i}$ computed by Eq. 4. In this case, we set the number of memory units $m$ to 10 .

From Figure 4, we observe that our memory units perform differently given different types of movies, which may illustrate that each memory unit in the memory network can represent one type of the user interest. For example, the Three Colors trilogy has quite similar attention weights in the memory network, since these three movies are loosely based on three political ideals in the motto of the French Republic. Die Hard is an action thriller movie, which is distinct from any other movies in the case study, explaining why it has a different weight pattern.

\section{Conclusion}

In this paper, we propose a memory augmented graph neural network (MA-GNN) for sequential recommendation. MAGNN applies a GNN to model items' short-term contextual information, and utilize a memory network to capture the long-range item dependency. In addition to the user interest modeling, we employ a bilinear function to model the feature correlations between items. Experimental results on five real-world datasets clearly validate the performance advantages of our model over many state-of-the-art methods and demonstrate the effectiveness of the proposed modules.

\section{References}

Battaglia, P. W.; Hamrick, J. B.; Bapst, V.; et al. 2018. Relational inductive biases, deep learning, and graph networks. CoRR abs/1806.01261. 
Belletti, F.; Chen, M.; and Chi, E. H. 2019. Quantifying long range dependence in language and user behavior to improve RNNs. In $K D D$.

Chen, X.; Xu, H.; Zhang, Y.; Tang, J.; Cao, Y.; Qin, Z.; and Zha, H. 2018. Sequential recommendation with user memory networks. In WSDM.

Cheng, C.; Yang, H.; Lyu, M. R.; and King, I. 2013. Where you like to go next: Successive point-of-interest recommendation. In IJCAI.

Cho, K.; van Merrienboer, B.; Gülçehre, Ç.; Bahdanau, D.; Bougares, F.; Schwenk, H.; and Bengio, Y. 2014. Learning phrase representations using RNN encoder-decoder for statistical machine translation. In $E M N L P$.

Deshpande, M., and Karypis, G. 2004. Item-based top$N$ recommendation algorithms. ACM Trans. Inf. Syst. 22(1):143-177.

Harper, F. M., and Konstan, J. A. 2016. The movielens datasets: History and context. TiiS 5(4):19:1-19:19.

He, R., and McAuley, J. J. 2016a. Fusing similarity models with Markov chains for sparse sequential recommendation. In ICDM.

He, R., and McAuley, J. J. 2016b. Ups and downs: Modeling the visual evolution of fashion trends with one-class collaborative filtering. In $W W W$.

He, X.; Liao, L.; Zhang, H.; Nie, L.; Hu, X.; and Chua, T. 2017. Neural collaborative filtering. In $W W W$.

He, R.; Kang, W.; and McAuley, J. J. 2017. Translationbased recommendation. In RecSys.

Hidasi, B., and Karatzoglou, A. 2018. Recurrent neural networks with top-k gains for session-based recommendations. In CIKM.

Hidasi, B.; Karatzoglou, A.; Baltrunas, L.; and Tikk, D. 2016. Session-based recommendations with recurrent neural networks. In ICLR (Poster).

Hochreiter, S., and Schmidhuber, J. 1997. Long short-term memory. Neural Computation 9(8):1735-1780.

Hu, Y.; Koren, Y.; and Volinsky, C. 2008. Collaborative filtering for implicit feedback datasets. In ICDM, 263-272.

Huang, J.; Zhao, W. X.; Dou, H.; Wen, J.; and Chang, E. Y. 2018. Improving sequential recommendation with knowledge-enhanced memory networks. In SIGIR.

Kang, W., and McAuley, J. 2018. Self-attentive sequential recommendation. In ICDM.

Koren, Y. 2008. Factorization meets the neighborhood: a multifaceted collaborative filtering model. In $K D D$.

$\mathrm{Li}, \mathrm{X}$., and She, J. 2017. Collaborative variational autoencoder for recommender systems. In $K D D$.

Li, J.; Ren, P.; Chen, Z.; Ren, Z.; Lian, T.; and Ma, J. 2017. Neural attentive session-based recommendation. In CIKM.

Lian, J.; Zhou, X.; Zhang, F.; Chen, Z.; Xie, X.; and Sun, G. 2018. xdeepfm: Combining explicit and implicit feature interactions for recommender systems. In $K D D$.

Liu, Q.; Zeng, Y.; Mokhosi, R.; and Zhang, H. 2018. STAMP: short-term attention/memory priority model for session-based recommendation. In $K D D$.
Ma, C.; Zhang, Y.; Wang, Q.; and Liu, X. 2018. Point-ofinterest recommendation: Exploiting self-attentive autoencoders with neighbor-aware influence. In CIKM.

Ma, C.; Kang, P.; Wu, B.; Wang, Q.; and Liu, X. 2019. Gated attentive-autoencoder for content-aware recommendation. In WSDM.

Miller, A. H.; Fisch, A.; Dodge, J.; Karimi, A.; Bordes, A.; and Weston, J. 2016. Key-value memory networks for directly reading documents. In $E M N L P$.

Ning, X.; Desrosiers, C.; and Karypis, G. 2015. A comprehensive survey of neighborhood-based recommendation methods. In Recommender Systems Handbook. Springer. 37-76.

Pan, R.; Zhou, Y.; Cao, B.; Liu, N. N.; Lukose, R. M.; Scholz, M.; and Yang, Q. 2008. One-class collaborative filtering. In ICDM.

Rendle, S.; Freudenthaler, C.; Gantner, Z.; and SchmidtThieme, L. 2009. BPR: Bayesian personalized ranking from implicit feedback. In UAI.

Rendle, S.; Freudenthaler, C.; and Schmidt-Thieme, L. 2010. Factorizing personalized Markov chains for nextbasket recommendation. In $W W W$.

Sukhbaatar, S.; Szlam, A.; Weston, J.; and Fergus, R. 2015. End-to-end memory networks. In NIPS.

Tang, J., and Wang, K. 2018. Personalized top-n sequential recommendation via convolutional sequence embedding. In WSDM.

Tran, T.; Liu, X.; Lee, K.; and Kong, X. 2019. Signed distance-based deep memory recommender. In $W W W$.

Vaswani, A.; Shazeer, N.; Parmar, N.; Uszkoreit, J.; Jones, L.; Gomez, A. N.; Kaiser, L.; and Polosukhin, I. 2017. Attention is all you need. In NIPS.

Wan, M., and McAuley, J. J. 2018. Item recommendation on monotonic behavior chains. In RecSys.

Xu, C.; Zhao, P.; Liu, Y.; Sheng, V. S.; Xu, J.; Zhuang, F.; Fang, J.; and Zhou, X. 2019. Graph contextualized selfattention network for session-based recommendation. In $I J$ CAI.

Xue, H.; Dai, X.; Zhang, J.; Huang, S.; and Chen, J. 2017. Deep matrix factorization models for recommender systems. In IJCAI.

Yu, L.; Zhang, C.; Liang, S.; and Zhang, X. 2019. Multiorder attentive ranking model for sequential recommendation. In $A A A I$.

Zhang, J.; Shi, X.; King, I.; and Yeung, D. 2017. Dynamic key-value memory networks for knowledge tracing. In $W W W$. 